\title{
Membrane transport
}

\section{Insights into L-type heteromeric amino acid transporters}

\author{
Simon Newstead ${ }^{1}$ \\ ${ }^{1}$ Department of Biochemistry, University of Oxford, Oxford, OX1 3OU, UK.
}

Two recent cryo-EM structures of the human L-type heteromeric amino acid transporter, LAT1-CDg8hc, reveal surprising new insights into both amino acid transport in the human body and the roles of $\mathrm{CDg} 8 / 4 \mathrm{~F} 2 \mathrm{hc}$ as a cell surface antigen and trafficking chaperone.

Whilst amino acids are familiar to many of us as the building blocks of proteins, they also play essential roles in regulating cellular metabolism (Figure 1). Proliferating cells need additional amino acids as precursors in metabolic pathways and to stimulate the mechanistic target of rapamycin complex $1\left(\operatorname{mTOR}_{1}\right)$, which functions to increase anabolic processes ${ }^{1}$. Cytoplasmic amino acid pools are controlled by several families of plasma membrane amino acid transporters, making these proteins important regulators of cell growth, upregulated in fast proliferating cancer cells, and hence important targets in ongoing anti-cancer drug discovery programmes ${ }^{2}$.

Mammalian amino acid transporters are categorised into several transport systems based on their substrate selectivity and mechanism. Dietary amino acid uptake is predominantly carried out by so-called System-L transporters, which mediate $\mathrm{Na}^{+}$independent transport of large amino acids across the plasma membrane ${ }^{3}$. Among these, the L-type amino acid transporter 1 ( $\left.\mathrm{LAT}_{1} ; \mathrm{SLC}_{7} \mathrm{~A}_{5}\right)$ has attracted the largest interest, due to the prominent role this member plays stimulating cancer cell growth through the uptake of L-leucine 4 , supplying thyroid hormones to developing foetuses and transporting L-3,4-dihydroxyphenylalanine (LDOPA) and other neurological drugs, across the blood brain barrier ${ }^{5}$. As a subunit of a heteromeric amino acid transporter (HAT), LAT1, referred to as the 'light' subunit associates with a second protein, called $4 \mathrm{~F}_{2} \mathrm{hc}$ or $\mathrm{CD} 98 \mathrm{hc}\left(\mathrm{SLC}_{3} \mathrm{~A} 2\right)$, referred to as the 'heavy' subunit. 
CDg8hc, with molecular weight of $85 \mathrm{kDa}$ for the mature form, comprises a single transmembrane domain and a large extracellular domain, which is heavily glycosylated ${ }^{6}$.

For several years our understanding of the structure of the LAT1-CDg8hc heteromeric complex have come from crystal structures of distantly related bacterial homologues of the transport domain $7^{-11}$ and an isolated crystal structure of the glycoprotein, minus the transmembrane segment ${ }^{6}$. Several key questions relating the function of mammalian LAT1 have therefore remained unanswered, such as how the light and heavy subunits interact in the membrane, the structural basis for amino acid specificity and an accurate structural model of the binding site to advance structure and computational based inhibitor/drug design.

Now, using single particle cryo-electron microscopy (cryo-EM), two groups have independently reported the structures of the human LAT1-CDg8hc complex ${ }^{12}$ (plus NSMB reference). LAT1 is captured in an inward open conformation, with the binding site accessible to the inside of the cell (Figure 2A). Nureki and colleagues report a structure in complex with two anti-CDg8hc antibodies that are currently being evaluated as anticancer agents. Zhou and colleagues report the complex with a competitive inhibitor $\mathrm{BCH}$, which functions as an anticancer molecule in tissue culture ${ }^{13}$. Both studies shed valuable insights into ligand recognition and potential structure-guided drug design, for either LAT1 inhibition or improved, LAT1-mediated transport through blood-brain barrier.

Do these structures explain the specificity for large neutral amino acids in LAT1? Nureki and colleagues noticed a distal pocket that extends out from the central binding site, which they speculate could accommodate the longer side chains of large, branched chained amino acids, such as L-leucine (Figure 2B). Indeed, this extension of the binding site is not present in the recent crystal structures of two closely related bacterial homologues, which do not transport L-leucine ${ }^{10,11}$, supporting a role for the distal pocket in amino acid selectivity. Can the structures explain why $\mathrm{BCH}$ is a competitive inhibitor of LAT1? Possibly. It is interesting to note that $\mathrm{BCH}$, unlike the substrates in relevant structures of related transporters, is not contacting a conserved tyrosine $\mathrm{Y}_{289}$ on $\mathrm{TM} 7$. It is possible, that without a direct interaction 
to this side chain, $\mathrm{BCH}$ is unable to trigger reorientation of the binding site and drive transport.

One of the most exciting aspects to emerge from these structures is our first glimpse of the interaction between a transporter and an ancillary subunit. There are several members of the $\mathrm{SLC}_{7}$ family that associate with $\mathrm{CD} 98 \mathrm{hc}\left(\mathrm{SLC}_{3} \mathrm{~A}_{2}\right)$. The function of $\mathrm{CD}_{9} 8 \mathrm{~h}$ c has been unclear, although it is known to function as a trafficking chaperone, escorting LAT1, through the secretory pathway to the plasma membrane and have a potential role in integrin signalling ${ }^{14,15}$. By reconstituting LAT1 in the absence of CDg8hc, however, Zhou and colleagues showed that LAT1 requires CDg8hc to function. Both teams also identified similar sets of interactions between the glycoprotein and LAT1. The structures however, are largely silent on why $L A T 1$ requires $C D g 8 h$ c to function. Perhaps the answer lies in the additional capacity of the heteromeric complex to bind lipids. Previous work had identified a requirement for cholesterol for the correct functioning of the LAT1-CDg8hc complex ${ }^{16}$. Both structures reveal that the interaction between $\mathrm{LAT}_{1}$ and $C D 98 \mathrm{hc}$ creates a lipid binding site within the membrane (Figure $2 \mathrm{C}$ ). Nureki and colleagues observe a cholesterol molecule bound in this pocket, whereas Zhou and colleagues identify two lipid-like densities that are modelled as phosphatidic acids. Lipids are emerging as important components of oligomeric interfaces in solute carrier transporters ${ }^{17}$. Of note is that the polar head groups of the phosphatidic acids interact with a conserved arginine of CDg8hc, mutation of which abolished transport function in LAT1. Clearly the ordering of lipids in this region of the heteromeric complex is important for transport function, although a more mechanistic interpretation will require additional studies.

Finally, the new structures reveal how two previously identified antibodies, HBJ127 and MEM108, which have anti-tumour activity in cell culture ${ }^{18}$, recognise their epitopes on CDg8hc. A key insight from these structures was that both antibodies bind to distinct epitopes on the glycoprotein, which do not interfere with the formation of the heterocomplex. As such, it now seems likely these antibodies function through antibodydependent cellular toxicity, rather than through inhibiting the transporter. 
Amino acid transport plays a fundamental role in cellular physiology, yet our understanding is still limited to largely bacterial homologues. The structures reported by Nureki and Zhou represent the first structures of human amino acid exchangers and hopefully are the harbingers of more structure-function studies into this physiologically and medically important family of solute carriers.

Funding provided by Wellcome (102890/Z/13/Z \& 215519/Z/19/Z).

\section{References.}

1. Efeyan, A., Comb, W.C. \& Sabatini, D.M. Nutrient-sensing mechanisms and pathways. Nature 517, 302-10 (2015).

2. Kandasamy, P., Gyimesi, G., Kanai, Y. \& Hediger, M.A. Amino acid transporters revisited: New views in health and disease. Trends Biochem Sci 43, 752-789 (2018).

3. Fotiadis, D., Kanai, Y. \& Palacin, M. The SLC3 and SLC7 families of amino acid transporters. Mol Aspects Med 34, 139-58 (2013).

4. Yanagida, O. et al. Human L-type amino acid transporter 1 (LAT1): characterization of function and expression in tumor cell lines. Biochim Biophys Acta 1514, 291-302 (2001).

5. del Amo, E.M., Urtti, A. \& Yliperttula, M. Pharmacokinetic role of L-type amino acid transporters LAT1 and LAT2. European journal of pharmaceutical sciences : official journal of the European Federation for Pharmaceutical Sciences 35, 161-174 (2008).

6. Fort, J. et al. The structure of human $4 \mathrm{~F} 2 \mathrm{hc}$ ectodomain provides a model for homodimerization and electrostatic interaction with plasma membrane. $J$ Biol Chem 282, 31444-52 (2007).

7. Fang, Y. et al. Structure of a prokaryotic virtual proton pump at $3.2 \mathrm{~A}$ resolution. Nature 460, 1040-1043 (2009).

8. Ma, D. et al. Structure and mechanism of a glutamate-GABA antiporter. Nature 483, 632-6 (2012).

9. Shaffer, P.L., Goehring, A., Shankaranarayanan, A. \& Gouaux, E. Structure and mechanism of a Na+-independent amino acid transporter. Science (New York, NY) 325, 1010-1014 (2009).

10. Jungnickel, K.E.J., Parker, J.L. \& Newstead, S. Structural basis for amino acid transport by the CAT family of SLC7 transporters. Nat Commun 9, 550 (2018).

11. Errasti-Murugarren, E. et al. L amino acid transporter structure and molecular bases for the asymmetry of substrate interaction. Nat Commun 10, 1807 (2019).

12. Yan, R., Zhao, X., Lei, J. \& Zhou, Q. Structure of the human LAT1-4F2hc heteromeric amino acid transporter complex. Nature 568, 127-130 (2019).

13. Kim, C.S. et al. BCH, an inhibitor of system $\mathrm{L}$ amino acid transporters, induces apoptosis in cancer cells. Biological \& pharmaceutical bulletin 31, 1096-1100 (2008). 
14. Palacin, M. \& Kanai, Y. The ancillary proteins of HATs: SLC3 family of amino acid transporters. Pflugers Arch 447, 490-4 (2004).

15. Boulter, E. et al. Cell metabolism regulates integrin mechanosensing via an SLC3A2dependent sphingolipid biosynthesis pathway. Nat Commun 9, 4862 (2018).

16. Dickens, D. et al. Modulation of LAT1 (SLC7A5) transporter activity and stability by membrane cholesterol. Sci Rep 7, 43580 (2017).

17. Bolla, J.R., Agasid, M.T., Mehmood, S. \& Robinson, C.V. Membrane Protein-Lipid Interactions Probed Using Mass Spectrometry. Annu Rev Biochem (2019).

18. Hayes, G.M. et al. Antitumor activity of an anti-CD98 antibody. Int J Cancer 137, 71020 (2015).

Figure Legends.

Figure 1. Physiological role of LAT1-CDg8hc heteromeric amino acid transporter. LAT1 regulates $\mathrm{mTORC}_{1}$ activity by controlling cytoplasmic concentrations of neutral branched chain amino acids (NBCAA) at the plasma membrane of eukaryotic cells. Several strategies are being developed to inhibit LAT1 in an effort to develop new cancer therapies. These involve either antibodies targeted to CDg8hc, or small molecule inhibitors of LATı transport.

Figure 2. Structure of LAT1-CD98hc complex. (A) Cryo-EM structure of LAT1 (PDB:6IRT).

(B) Zoomed in view of the binding site, where a competitive inhibitor, $\mathrm{BCH}$, was captured, and the identification of a distal pocket, which is proposed to contribute to amino acid selectivity. View looking up into the binding pocket from the cytoplasm. (C) Formation of the heteromeric complex leads to the creation of a novel lipid binding site, which can accommodate both cholesterol and phosphatidic acid as observed in both structures. View as in $(A)$. 
Figure 1

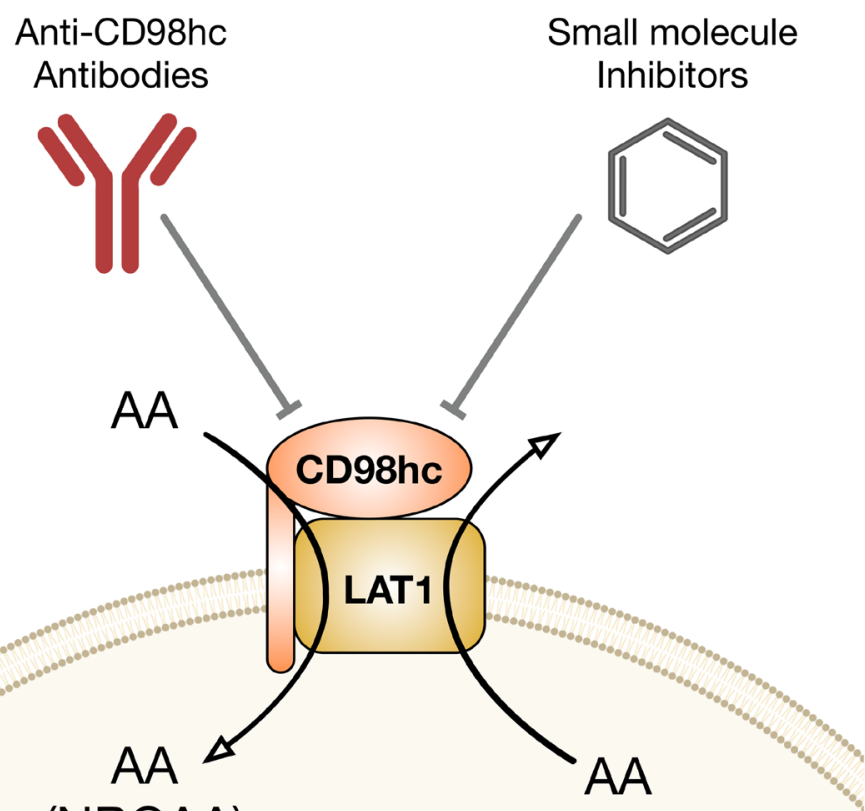

(NBCAA)

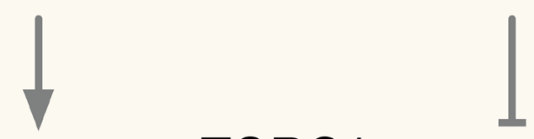

mTORC1

(Cell proliferation) 
Figure 2

A

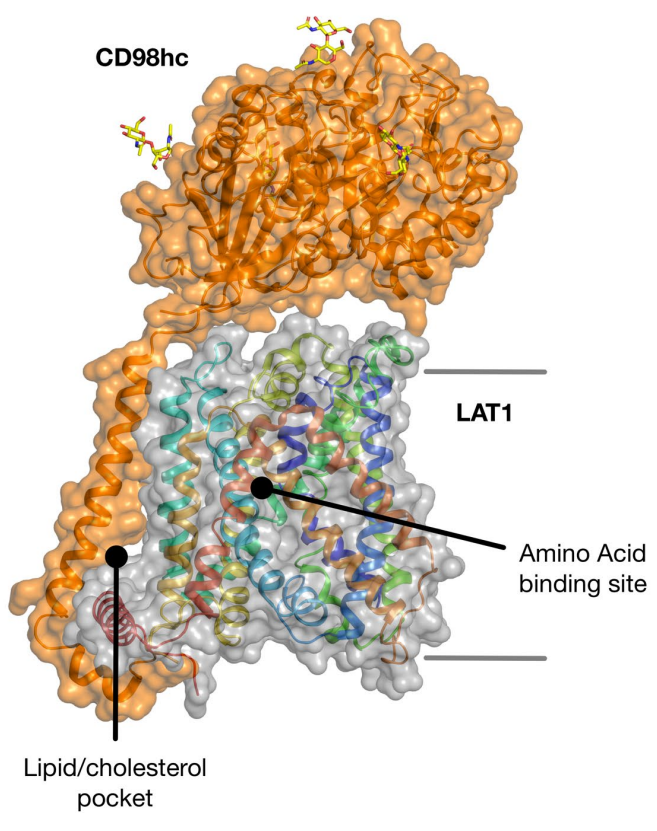

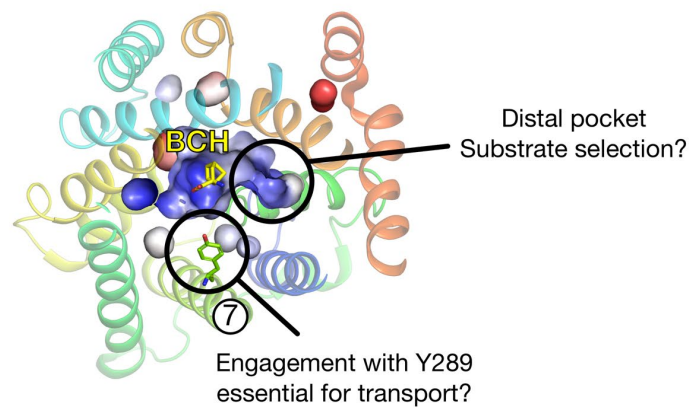

C

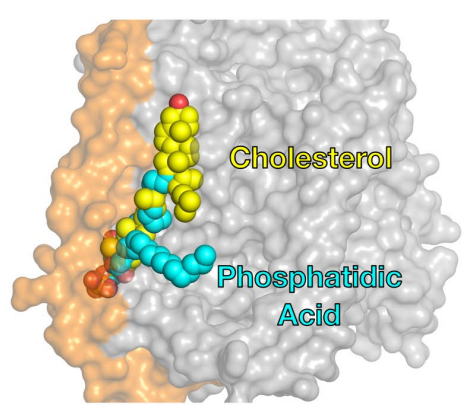

